\title{
Motion of a relativistic particle and the vacuum*
}

\author{
Volodymyr Krasnoholovets
}

Institute of Physics, National Academy of Sciences, Prospect Nauky 46, UA-03650 Kyïv 39, Ukraine

Received 24 May 1994

\begin{abstract}
A vacuum medium model is advanced. The motion of a relativistic particle in relation to its interaction with the medium is discussed. It is predicted that elementary excitations of the vacuum, called "inertons," should exist. The equations of the particle path in Euclidean space are derived. The motion is marked by the basic quantum mechanical relations $E=h \nu$ and $M v_{0}=h / \lambda$ (here, $\lambda$ is the amplitude of spatial oscillations of the particle along the trajectory, i.e., the interval at which the velocity of the particle is periodically altered from $v_{0}$ to 0 and then from 0 to $v_{0} ; \nu$ is the frequency of these oscillations). Analysis is performed on the transition to wave mechanics where $\lambda$ manifests itself as the de Broglie wavelength, and $\nu$ is the distinctive frequency of the "particle-wave". A prerequisite for the wave solution to be Lorentz-invariant is treated. A hypothesis for a plausible hydrodynamic description of the relativistic particle motion is covered.
\end{abstract}

Key words: geometry of space, metric tensor, relativity, physical vacuum, hidden variables, elementary particles, hypothetical particles, quantum mechanics

*Physics Essays, 10, no. 3, pp. 407-416 (1997) 


\section{Introduction}

Orthodox nonrelativistic quantum mechanics is well known ${ }^{(1)}$ to suffer from long-range action. Furthermore, nonrelativistic quantum mechanics is not Lorentz-invariant, although checking any theory for Lorentz-invariance is now considered to be the best guarantee of its reliability. Relativistic quantum mechanics does not suffer from the above difficulties. However, both these theories work very well in the area of microscopic phenomena, and experiment confirms this fact perfectly. In connection with this it can be assumed that a hidden mechanism uniting these two limiting cases should exist, and it may result in the universal quantum theory.

There are different ways of devising a solution to the problem. One way is based on the causal interpretation of the particle behaviour in the system. Causal (deterministic) concepts have been developed now in many papers (see, e.g., Refs. 2 to 6 ), yet modeling of possible structural peculiarities of the vacuum medium in which the particle moves is not presented in any of them. And some papers treat the vacuum space in a more or less explicit form as a discrete lattice $^{(7-11)}$, as a peculiar kind of a quantum crystal, ${ }^{(12)}$ or as a model of Planck aether $^{(13)}$.

In the preceding paper ${ }^{(14)}$ we have postulated a specific cellular structure for the physical vacuum and discussed deterministic dynamics of a nonrelativistic particle. In Ref. 14 an introduction of the potential of the particle's interaction with the vacuum showed that the motion of the particle is of an oscillating character: initially, at the section $\lambda / 2$ of the path, the vacuum medium knocks elementary excitations (inertons) out of the moving particle. They migrate ahead of the particle, and the velocity of the particle gradually decays to zero and, subsequently, at the next section $\lambda / 2$ of the path, the particle absorbs inertons, this time from behind, and the quantity of its velocity regains its original value $v_{0}$. As this takes place, the amplitude $\lambda$ of the particle's space oscillations is identified as the de Broglie wavelength.

The present paper develops the model described in Ref. 14 when the relativistic spinless particle is in motion.

\section{Vacuum medium}

The fundamental notions of modern geometry (in coordinate approach) are as follows ${ }^{(15)}$ : (1) the point in space, (2) the vector of this point's motion velocity, (2) the line length along which the point moves. Some spaces (Euclidean, Riemannian, and others) are specified above all by their metric. In addition, we introduce the discreteness of space, dividing it into uniform cells the size of which is close to the value $10^{-28} \mathrm{~cm}$, as required by the grand unification of interactions (all types of interaction come together in this scale). Let us assume that each of these these cells contains a superparticle, which is in the degenerate state over all possible multiplets, since for each elementary particle there is a 
certain multiplet state. The degeneration of the superparticle may be released spontaneously or in response to the external factor as a result of which its volume changes and a certain symmetry appears, that is, an elementary particle is created. In the author's view, a change in volume of the initial superparticle is associated with the emergence of the mass of the particle at rest. Therefore, the particle represents a defect in the homogeneous discrete vacuum space; hence the space around this defect must be rendered somewhat deformed: the neighboring cells are subjected to deformation that may be identified with the gravitational potential appearing in the space surrounding the particle.

The motion of the physical "point" (particle cell) in the entirely packed discrete space is accompanied by the interaction with the "points" of the space (superparticles cells), giving rise to excitations in neighboring superparticles. As the particle moves, it constantly emits and absorbs excitations (inertons), but in distinction to the real motion of the particle, the motion of inertons is different: they migrate by a relay mechanism, from superparticle to superparticle. Since inertons are elementary excitations of the vacuum medium, their velocity, at least the initial velocity, is of the order or equal to the speed of light $c$, the velocity of the particle $v_{0}<c$. Let us consider the motion of such system in the relativistic approximation.

\section{$3 \quad$ Lagrangian and the particle trajectory}

In Ref. 14 we proceeded from the modernized classical nonrelativistic Lagrangian of the particle of the type

$$
L_{\text {nonrel. }}=\frac{1}{2} g_{i j} \dot{X}^{i} \dot{X}^{j}+U(X, x, \dot{x}),
$$

where the function $U$ accounted for the potential energy of the interaction between the particle and inertons and the kinetic energy of inertons (hereinafter the vectors $X$ and $\dot{X}$ pertain to the particle, the vectors $x$ and $\dot{x}$ pertain to inertons). On examination of the relativistic particle, we shall proceed in a similar fashion, that is, in the classical relativistic Lagrangian

$$
L_{\text {rel. }}=-M_{0} c^{2} \sqrt{1-v_{0}^{2} / c^{2}}
$$

we shall substitute

$$
v_{0}^{2} \longrightarrow\left[g_{i j} \dot{X}^{i} \dot{X}^{j}+U(X, x, \dot{x})\right] / g .
$$

The modernized relativistic Lagrangian of the particle, in view of the interaction with inertons, is written in an explicit form as (cf. with Ref. 14):

$$
\mathcal{L}=-g c^{2}\left\{1-\frac{1}{g c^{2}}\left[g_{i j} \dot{X}^{i}(t) \dot{X}^{j}(t)+\sum_{r=0}^{N-1} \widetilde{g}_{i j}^{(r)} \dot{x}_{(r)}^{i}\left(t_{(r)}\right) \dot{x}_{(r)}^{j}\left(t_{(r)}\right)\right.\right.
$$




$$
\begin{gathered}
-\sum_{r=0}^{N-1} \frac{2 \pi}{T_{(r)}} \delta_{t-\Delta t_{(r)}, t_{(r)}}\left(X^{i}(t) \sqrt{g_{i s}\left(\widehat{A}^{-1} \widetilde{g}_{s j}^{(r)}\right)_{0}} \dot{x}_{(r)}^{j}\left(t_{(r)}\right)\right. \\
\left.\left.\left.+\left.\dot{X}^{i}(t)\right|_{t=0} \sqrt{g_{i s}\left(\widehat{A}^{-1} \widetilde{g}_{s j}^{(r)}\right)_{0}} x_{(r)}^{j}\left(t_{(r)}\right)\right)\right]\right\}^{1 / 2} .
\end{gathered}
$$

Here, $g_{i j}$ are components of the metric tensor produced by the particle in the three-dimensional space; along the trajectory of the particle $g_{i j}=$ const $\delta_{i j}$ and $g=g_{i j} \delta^{i j}$ is the convolution of the tensor; $\widetilde{g}_{i j}^{(r)}\left(x_{(r)}\right)$ are components of the metric tensor of the $r$ th inerton in the three-dimensional space, along the trajectory of the $r$ th inerton the tensor $\widetilde{g}_{i j}^{(r)}$ is supposed to be locally equal to const $\delta_{i j}$ (index $r$ is enclosed in parentheses to distinguish it from indices $i, j$ and $s$ that describe tensor and vector quantities); $1 / T_{(r)}$ is the frequency of collisions of the particle with the $r$ th inerton, $N$ is the number of inertons emitted by the particle; $\delta_{t-\Delta t_{(r)}, t_{(r)}}$ is Kroneker's symbol that provides the agreement between the proper time $t$ of the particle and $t_{(r)}$ that of the $r$ th inerton at the instant of their collision $\left(\Delta t_{(r)}\right.$ is the time interval after expiry of which, measuring from the initial moment $t=0$, the moving particle emits an inerton). The operator $\hat{A}^{-1}$ allows for the motion of the metric in every inerton at the instant of its emission (absorption) by the particle: $\widetilde{g}_{s j}^{(r)} \rightarrow \widetilde{g}_{s+u, j}^{(r)}$ (with regard to cyclic permutation, indices $s, j$ and $u$ take on values 1,2 and 3 ), that is, it shifts the emitted inerton to the trajectory distinct from the path of the particle. As the proper time $t$ of the particle is expressed through the proper time $t_{(r)}$ of the $r$ th excitation, $t=t_{(r)}+\Delta t_{(r)}$ (see Ref. 14), it suffices to write Euler-Lagrange equations for the particle and the $r$ th inerton in terms of one of these two time parameters, for instance, via $t_{(r)}$ :

$$
d\left(\partial \mathcal{L} / \partial \dot{Q}^{k}\right) / d t_{(r)}-\partial \mathcal{L} / \partial Q^{k}=0
$$

where for the particle

$$
Q^{k} \equiv X^{k}\left(t_{(r)}+\Delta t_{(r)}\right)
$$

and for the $r$ th inerton

$$
Q^{k} \equiv x_{(r)}^{k}\left(t_{(r)}\right) .
$$

When moving, the particle constantly exchanges the energy and momentum with the ensemble of inertons. In this case, the laws of conservation of the total energy $E$ and the total momentum $p_{0}$ for the particle-inertons' ensemble system should be obeyed along the path $l$. The constants $E$ and $p_{0}$ are obviously the initial values for the particle at the trajectory [at initial instant $t=0$, the Lagrangian (4) still retains its classical form: expression (2), in terms of which quantities $E$ and $p_{0}$ are derived using the standard method; see, e.g., Refs. 16, 17]. Since the expression under the radical symbol in the Lagrangian (4) is constant, we may treat the time $t$ as the parameter proportional to the natural $l$ ( evidence for this statement, for instance, for the Lagrangian $L=\sqrt{g_{i j} \dot{X}^{i} \dot{X}^{j}}=$ const, see Ref. 18). However, as time is considered to be the parameter accordant with the particle path length, $t \propto l$ (for inertons 
$t_{(r)} \propto l_{(r)}$, where $l_{(r)}$ is path length for $r$ th inerton), equations of extremals for the Lagrangian (4) are in complete agreement with the appropriate equations obtained in Ref. 14 for the nonrelativistic case (see Ref. 19, which shows identity of equations for extremals derived from the Lagrangians $L=g_{i j} \dot{X}^{i} \dot{X}^{j}$ and $L=\sqrt{g_{i j} \dot{X}^{i} \dot{X}^{j}}$, with the allowance made for a natural character of the parameter $t$ ).

Some details for solving equations of motion for the particle and inertons are adduced in Appendix. We shall present here the ultimate result true to one period $T_{(r)}$ of collisions, that is, the time interval between the moments of emission and absorption of the $r$ th inerton by the particle. If the particle moves along the axis $X^{1} \equiv X$, then the motion of inertons is defined by the coordinates longitudinal and transversal to the $X$-axis, that is, $x_{(r)}^{\|}$and $x_{(r)}^{\perp}=$ $\left[\left(x_{(r)}^{2}\right)^{2}+\left(x_{(r)}^{3}\right)^{2}\right]^{1 / 2}$, respectively (hereinafter, we omit the parentheses for indices $r$ ):

$$
\begin{gathered}
\dot{x}_{r}^{\perp}\left(t_{r}\right)=c \cos \left(\pi t_{r} / T_{r}\right) ; \\
x_{r}^{\perp}\left(t_{r}\right)=\frac{\Lambda}{\pi} \sin \left(\pi t_{r} / T_{r}\right) ; \\
\Lambda_{r}=c T_{r} .
\end{gathered}
$$

For the longitudinal coordinate

$$
\dot{x}_{r}^{\|}=\text {const }=3 v_{0 r} / 2 \pi
$$

where

$$
v_{0 r}=v_{0}[1-\sin (r \pi / 2 N)],
$$

$v_{0}$ is the initial velocity of the particle at the moment $t_{r}=t=0$. In formulas (6) to (9), the variable $t_{r}$ determines the proper time of the $r$ th inerton within the time interval $T_{r}$. For the particle the solutions take the form

$$
\begin{gathered}
\dot{X}\left(t_{r}\right)=v_{0 r}\left[1-\sin \left(\pi t_{r} / T_{r}\right)\right] ; \\
X\left(t_{r}\right)=v_{0 r} t_{r}+\frac{\lambda_{r}}{\pi}\left[\cos \left(\pi t_{r} / T_{r}\right)-1\right] ; \\
\lambda_{r}=v_{0 r} T_{r} .
\end{gathered}
$$

As seen above, the solutions (11) and (12) are written in terms of the proper time of the $r$ th inerton. In Ref. 14 solutions (6), (7), (9), (11) and (12) were lengthened for the next half-periods of oscillations $n T_{r}$, where $n$ is the number of the spatial oscillation of the particle along the trajectory from the initial point of its motion. However, the quasicontinuous time parameter $t_{r n}$ has been introduced for this purpose. In the case of the relativistic Lagrangian (4), the parameter $t$ is regarded as being natural, proportional to the trajectory length $l$; hence this parameter should necessarily be continuous. This requirement may be readily satisfied, if to the initial condition $\left.\dot{X}\right|_{t=0}=v_{0}$ [see formula (A11)] we 


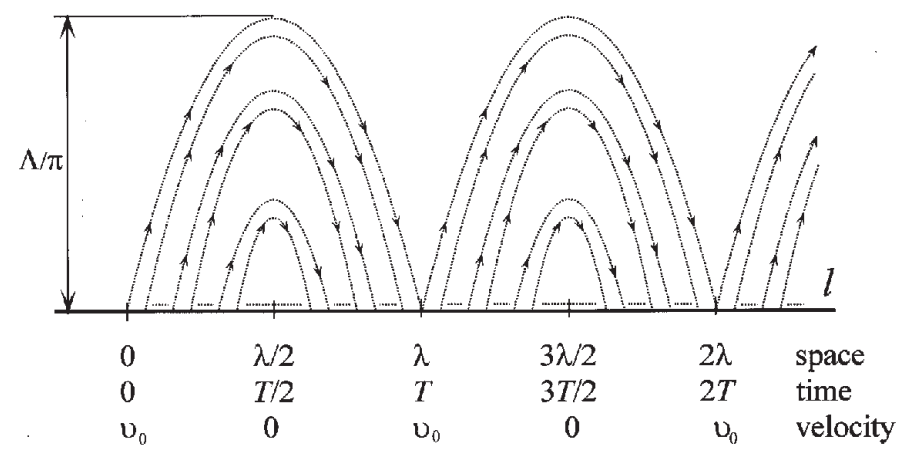

Figure 1: The schematic representation of the motion trajectories of the particle (along its path $l$ ) and attending inertons.

add another requirement in accordance with which the quantity of the particle velocity for the $n$th time period of collisions $T$ resumes its initial value, that is,

$$
\left.\dot{X}\right|_{t=0}=\left.\dot{X}\right|_{t=n T}=v_{0}, \quad n=1,2,3, \ldots
$$

[with arbitrary $t$, variable $\dot{X}(t) \geq 0$ ]. At this condition the solutions from (11) to (13) as functions of the proper time $t$ of the particle assume the form (by the way, true and, besides, more attractive one for nonrelativistic case):

$$
\begin{gathered}
\dot{X}(t)=v_{0}(1-|\sin (\pi t / T)|) ; \\
X(t)=v_{0} t+\frac{\lambda}{\pi}\left\{(-1)^{[t / T]} \cos (\pi t / T)-(1+2[t / T])\right\} ; \\
\lambda=v_{0} T .
\end{gathered}
$$

Equation (11) has been integrated over each $n$th interval, so the solutions for all $n$ intervals have been united. In this case $X$ as a function of $t$ in $(15 b)$ is the continuous and positive value with the initial condition $X(0)=0$. The notation $[t / T]$ in $(15 b)$ means an integral part of the integer $t / T$.

Motion trajectories of the particle and surrounding inertons are illustrated schematically in Fig. 1. As can be seen, the path of the particle may be divided into equal sections of length $\lambda$, even though the parameter $t$ is continuous along the trajectory. The parameter $\lambda$ should be regarded as the space period or as the amplitude of the particle's spatial oscillations. It is of interest that in conformity with correlation (16), the time half-period of oscillations $T$ may be written as $T=\lambda / v_{0}$ (for inertons $T_{r}=\Lambda_{r} / c$ ). Thus the quantity $T$ is the natural parameter at the length of the spatial period $\lambda$ for the particle, much as its proper time $t$ is the natural parameter for the entire trajectory, $t=l / v_{0}$. The quantity $T_{r}$ may be viewed as the natural parameter at the length of the spatial period $\Lambda_{r}$ of the $r$ th inerton. However, for each portion the proportionality 
coefficients between $T$ and $\lambda$ for the particle as well as $T_{r}$ and $\Lambda_{r}$ for inertons differ essentially and are equal to $1 / v_{0}$ and $1 / c$, respectively.

\section{Wave relativistic mechanics}

If the ensemble of inertons is considered as the whole object, an inerton cloud with the effective mass at rest $m_{o}$, then the original Lagrangian should be accordingly altered. In Euclidean space it may be presented as

$$
\begin{aligned}
L= & -M_{0} c^{2}\left\{1-\frac{1}{M_{0} c^{2}}\left[M_{0} \dot{X}^{2}+m_{0} \dot{x}^{2}\right.\right. \\
& \left.\left.-\frac{2 \pi}{T} \sqrt{m_{0} M_{0}}\left(X \dot{x}+v_{0} x\right)\right]\right\}^{1 / 2} .
\end{aligned}
$$

The function (17) describes the particle with the mass at rest $M_{0}$ that moves along the $X$-axis with the velocity $\dot{X}$ ( $v_{0}$ is initial velocity); $x$ is the distance between the inerton cloud and the particle, $\dot{x}$ is the velocity of cloud in the

frame of reference connected with the particle, and $1 / T$ is the frequency of collisions between the particle and inerton cloud. We believe that $x$ and $\dot{x}$ are functions of the proper time $t$ of the particle.

Euler-Lagrange equations for the Lagrangian (17) are reduced to the following system [for this purpose relationship $M_{0} / m_{0}=c^{2} / v_{0}^{2}$ from formula (A6) is used]

$$
\begin{gathered}
\ddot{X}+\frac{\pi}{T} \frac{v_{0}}{c} \dot{x}=0 ; \\
\ddot{x}-\frac{\pi}{T} \frac{c}{v_{0}}\left(\dot{X}-v_{0}\right)=0 .
\end{gathered}
$$

The solutions $x(t)$ and $\dot{x}(t)$ obtained from Eqs.(18), (19) have the form

$$
\begin{gathered}
x=\frac{\Lambda}{\pi}|\sin (\pi t / T)|, \\
\dot{x}=c(-1)^{[t / T]} \cos (\pi t / T)
\end{gathered}
$$

where $\Lambda$ is the amplitude of the inerton cloud oscillation. The solutions $X(t)$ and $\dot{X}(t)$ comply with expressions (15). Solutions of $X, \dot{X}$ and $x, \dot{x}$ are shown in Fig. 2. Notice that for the decision of Eqs. (18), (19) initial conditions $x(0)=X(0)=0$, condition (14), inequalities $x, X, \dot{X} \geq 0$ and $c \geq \dot{x} \geq-c$ have been taken into account.

While substituting

$$
\dot{\widetilde{x}}=\dot{x}-\frac{\pi}{T} \sqrt{M_{0} / m_{0}} X
$$

the Lagrangian (17) is reduced to the canonical form on variables for the particle:

$$
L=-M_{0} c^{2}\left\{1-\frac{1}{M_{0} c^{2}}\left[M_{0} \dot{X}^{2}-M_{0}(2 \pi / 2 T)^{2} X^{2}\right.\right.
$$



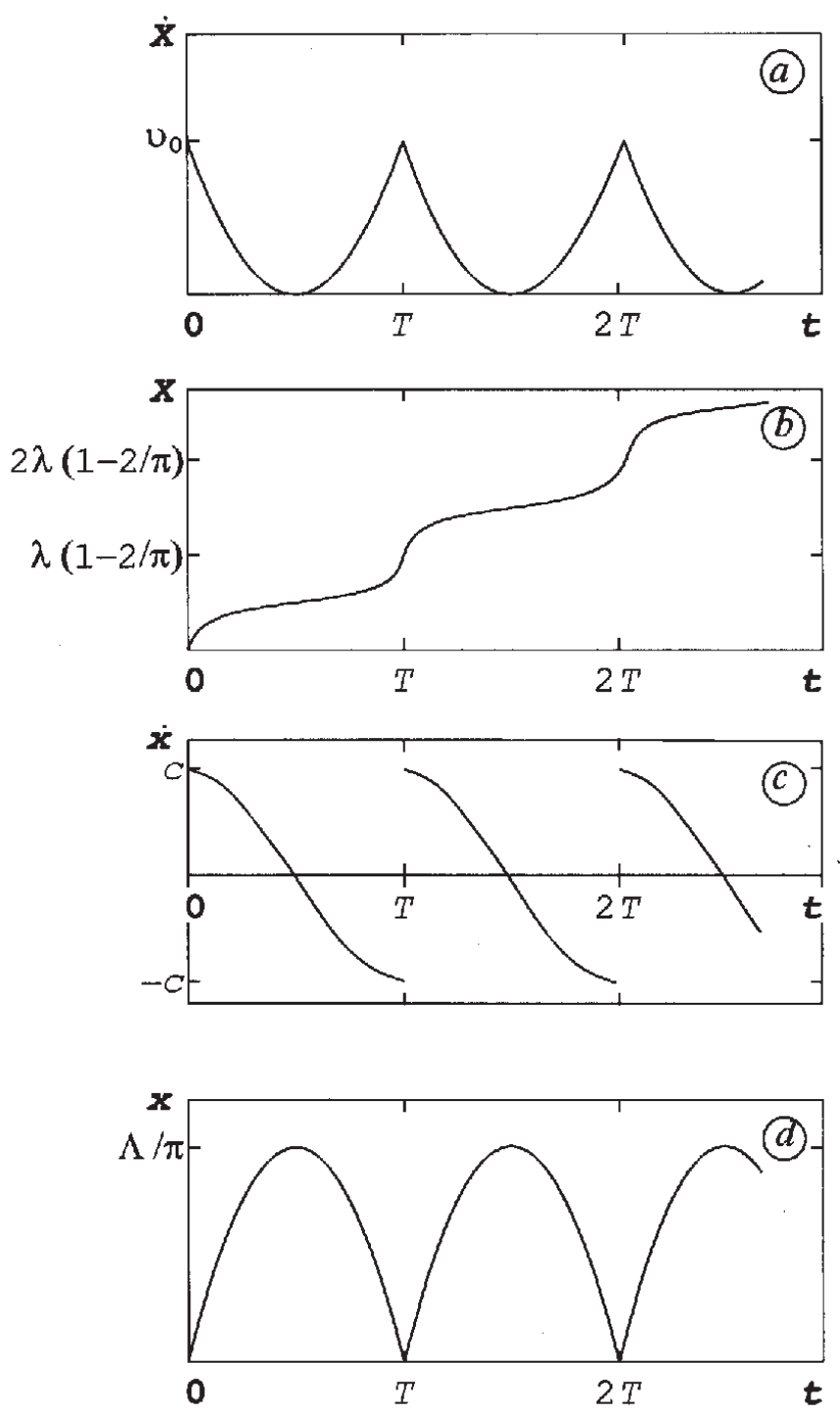

Figure 2: The schematic representation of the solutions of Eqs. (15) for the particle $(a, b)$ and Eqs. (20) for the inerton cloud $(c, d)$. 


$$
\left.\left.+m_{0} \dot{\widetilde{x}}^{2}-\frac{2 \pi}{T} \sqrt{m_{0} M_{0}} v_{0} x\right]\right\}^{1 / 2} .
$$

As seen above, the first two terms in brackets under the radical in (22) describe the particle alone and comply with the kinetic and potential energies of the harmonic oscillator; the last two terms depict the effective kinetic and potential energies of the inerton cloud.

Let us introduce the Hamiltonian function conforming to the Lagrangian (22). In accordance with the definition

$$
H=\sum_{i} \dot{Q}_{i} \partial L / \partial \dot{Q}_{i}-L
$$

Therefore, for our case we have

$$
H=\dot{X} \partial L / \partial \dot{X}+\dot{\widetilde{x}} \partial L / \partial \dot{\widetilde{x}}-L ;
$$

hence for the function $L(22)$ we derive the corresponding Hamiltonian

$$
H=M(2 \pi / 2 T)^{2} X^{2}+M c^{2}+\pi \sqrt{m M_{0}} v_{0} x / T
$$

where

$$
M=M_{0} / \sqrt{1-v_{0}^{2} / c^{2}}, \quad m=m_{0} / \sqrt{1-v_{0}^{2} / c^{2}} .
$$

When deriving (24), we have paid attention to the fact that in the Lagrangian (22) the radical $\sqrt{\cdots}$ is const, equal to $\sqrt{1-v_{0}^{2} / c^{2}}$. On the other hand, in compliance with the derivation of momenta for the particle $p$ and of the inerton cloud $\widetilde{p}$, we obtain

$$
\begin{gathered}
p=\partial L / \partial \dot{X}=M \dot{X} \\
\widetilde{p}=\partial L / \partial \dot{\widetilde{x}}=m \dot{\widetilde{x}} .
\end{gathered}
$$

Thus with allowance made for the expressions (25), (26) and (22), the Hamiltonian (23) may be presented as

$$
H=p^{2} / M+\widetilde{p}^{2} / m+\left(M_{0} c\right)^{2} / M .
$$

Both presentations (24) and (27) of the relativistic Hamiltonian are equivalent. By combining (24) and (27), the following Hamiltonian is produced

$$
\begin{aligned}
H=p^{2} / 2 M+ & M(2 \pi / 2 T)^{2} X^{2} / 2+\left[(M c)^{2}+\left(M_{0} c\right)^{2}\right] / 2 M \\
& +\widetilde{p}^{2} / 2 m+\pi \sqrt{m M_{0}} v_{0} x / T .
\end{aligned}
$$

The first two terms in (28) describe the particle and represent the Hamiltonian of the harmonic oscillator; the third term is the renormalized energy of the particle at rest, and the last two terms are the kinetic and renormalized potential energies of the inerton cloud.

Let us separate out of (28) the effective Hamiltonian of the particle that describes its behavior relative to the inertia centre of the particle-inerton cloud system:

$$
H_{\text {eff }}=p^{2} / 2 M+M(2 \pi / 2 T)^{2} X^{2} / 2 .
$$


As done in Ref. 14, we now turn our attention to the function of the action. For a shortened action $S_{1}$ of the particle we have the Hamilton-Jacobi's equation:

$$
\left(\partial S_{1} / \partial X\right)^{2} / 2 M+M(2 \pi / 2 T)^{2} X^{2} / 2=E .
$$

For the action $S_{1}$ from Eq. (30) we acquire

$$
S_{1}=\int^{X} p d X=\int^{X} \sqrt{2 M\left[E-M(2 \pi / 2 T)^{2} X^{2} / 2\right]} d X .
$$

The solution for $X$ as the function of $t$ has the appearance (see, e.g., Refs. $20,21)$

$$
X=\sqrt{\frac{2 E}{M(2 \pi / 2 T)^{2}}} \sin (2 \pi t / 2 T) .
$$

The periodicity in the particle motion allows to pass on to the action-angle variables in Eq. (31) that depicts this motion. Hence for the increment of the particle action within the cyclic period $2 T$ we obtain

$$
J=\oint p d X=E \times 2 T .
$$

Allowing for an agreement between the constant $J$ and the initial kinetic energy of the particle $E=M v_{0}^{2} / 2$, expression (33) may be rewritten as

$$
J=M v_{0} \times v_{0} T=p_{0} \lambda
$$

where $p_{o}$ is the initial momentum, and, when deriving (34), the relation (16) is used. By assuming that $J=h$ ( $h$ is the Planck constant), we obtain the de Broglie relation (for relativistic particle) from (34):

$$
h / \lambda=p_{0} \equiv M v_{0} .
$$

According to our interpretation, in formula (35) $\lambda$ is the spatial period of the particle oscillations on the trajectory. Since $2 T$ is the cyclic period of the particle oscillations, then by introducing the appropriate frequency $\nu=1 / 2 T$ into (33) and assuming that $J=h$, we obtain another of the two main relations of quantum mechanics:

$$
E=h \nu .
$$

But it should be noted that the constant $E$ is consistent with the initial kinetic energy of the relativistic particle, $E=M v_{0}^{2} / 2$.

In configurational space where the solution (32) for $X$ is found, the variable $X$ is not restricted by the spatial period $\lambda$. Here, the trajectory of the particle is continuous and in the course of time the particle moves steadily away from the initial point $X=0$. Relations (35) and (36) admit a correlation between the particle that constantly moves along the $X$-axis of configurational space and a plane monochromatic wave that travels in the same direction and is marked 
by the frequency $E / h$ and wavelength $h / p_{0}$. This correlation is known ${ }^{(22)}$ (see also Ref. 14) to result in Schrödinger wave equation

$$
\left(\hbar^{2} / 2 M\right) \nabla^{2} \psi+E \psi=0
$$

where the wave function

$$
\psi=\psi_{0} \exp [2 \pi i(X / \lambda-\nu t)]
$$

\section{Discussion}

Schrödinger equation (37) is somewhat specific as it describes the behavior of the particle with the relativistic mass $M=M_{0} / \sqrt{1-v_{0}^{2} / c^{2}}$. In this equation, the momentum operator $-i \hbar \nabla$ and the eigenvalue $E$ are related to the relativistic momentum $M v_{0}$ and relativistic kinetic energy in the form of $M v_{0}^{2} / 2$, respectively. In the approximation $v_{0}^{2} / c^{2} \ll 1$, Eq. (37) reduces to a common nonrelativistic Schrödinger equation. Simply a part of the particle's total energy, namely, its kinetic component, $M v_{0}^{2} / 2$ appears in Eq. (37) due to the obtained correlation (36), where precisely the kinetic energy of the particle-inerton cloud system defines the frequency of the system's oscillations.

The equality $E=h \nu$ is formally postulated for the particle in quantum mechanics; both of the quantities $E$ and $\nu$ are indeterminate in this correlation: $E$ at times imply just the kinetic energy $M_{0} v_{0}^{2} / 2$ of the nonrelativistic particle (see, e.g., Fermi ${ }^{(23)}$ and Schiff( ${ }^{(24)}$ ) and at other times the total energy $M c^{2}$ (see, e.g., Schiff ${ }^{(25)}$ and Sokolov et al. $\left.{ }^{(26)}\right)$. De Broglie himself, having introduced the relation (36) for the particle, adhered to both of these contrary opinions relative to $E$ at different times (see Ref. 27 and comments by Lochak in it). But the fact that the proper frequency $\nu$ attributable to the moving particle cannot be determined by its total energy $M c^{2}$ may be supported by the argument that precisely this formula is applied for description of annihilation process. For instance, by annihilation of an electron and positron that have the same energies $E_{\text {tot }} \equiv M c^{2}$, the energy $h \nu$ of the two emitted photons is found precisely from the equation $2 E_{\text {tot }}=2 h \nu$. Therefore, it is not logical to ascribe the frequency $\nu=E_{\text {tot }} / h$ to the particle as this correlation automatically defines the particle instability in relation to its transmutation. At the same time, the above-obtained relation (36), where $E$ is simply the kinetic component $M v_{0}^{2} / 2$ of the total energy $M c^{2}$, is internally noncontradictory in the context of the proposed model.

We shall now highlight another important detail related to Eq. (37) or, more exactly, to the generalized wave equation

$$
\left(i \hbar \frac{\partial}{\partial t}+\frac{\hbar^{2}}{2 M} \nabla^{2}\right) \psi=0
$$

where the $\psi$-function is defined in (38). As mentioned above, time $t$ is the proper time of the particle proportional to the natural parameter $l$; namely, in the case 
of the Lagrangians (4) and (17) the proper time $t$ has been chosen in the form of $t=l / v_{0}$. In other words, we suppose that the form has been predetermined by the initial value of the particle velocity $v_{0}$. So the chosen proper time $t$ is the proper time along the world line $l$, and, according to definition (see, e.g. Refs. 28 and 29) these two parameters $(l$ and $t)$ should be invariant Lorentz transformations. The path is made up of identical sections of length $\lambda$; hence the invariance $\lambda$ follows from Lorentz invariance of the quantity $l$, and since the time half-period $T$ of the cycle of the particle is proportional to $\lambda$, that is, $T=\lambda / v_{0}$, then the quantity $T$ will also be invariant. Thus the variables $X$ and $t$ shared by the spatial $\lambda$ and time $T$ intervals, respectively, are Lorentz-invariant in the wave equation (39).

Each interaction geometrization stage in physics calls for a penetrating insight into physical processes of the phenomenon we attempt to formalize. Thus in solids and liquids relativistic effects apparently stem from a change in intermolecular and interatomic forces, and it is just this hidden dynamics that should be the basis for the formalism of special relativity.

Lorentz also indicated this possibility ${ }^{(30,31)}$. In 1902 and 1904 he proposed hypotheses on the change due to the motion of the intermolecular forces, geometry of the electron, and its mass in the aether. In 1913 Ehrenfest $^{(32)}$ analyzed views of Lorentz and Einstein (in 1905 the latter suggested ${ }^{(33)}$ that the speed of light in a vacuum is constant). Ehrenfest came to the conclusion that Einstein's special relativity, which denied the aether, leads to the same results as Lorentz's aether theory. In this case, he had stressed, such experimentum crucis which could solve the controversial question in favor of this or that theory cannot be realized. Nevertheless, as a consequence of the postulate of the universality of the speed of light $c$ and the principle of equivalence of an infinite multitude of inertial frames of reference forced out the notion of the orthodox aether. So the use of Lorentz kinematic transformations have been sufficient to give the final results.

These transformations are universal for all measured physical magnitudes (coordinate, time, electric and magnetic fields, etc.). Therefore, it really seemed quite natural to drop the notion of an aether medium with its inner deformations which are not measurable in principle. Why should this medium be introduced into the space between material objects, if any instrument by its nature is oriented only to a measurement of characteristics connected solely with those material objects? It was just special relativity that offered the clue to finding the change in physical magnitudes when an object moves, and a notion of an intermediate medium between objects is not necessary for this purpose.

This is undoubtedly so. However, on the other hand, all the modern significant achievements in the solid area are based on the noncausal and nonrelativistic, without Lorentz-invariance quantum mechanics. Thus for decades we found ourselves in a paradoxical situation because we must trust both the special theory of relativity, which has been substantiated by numerous experiments, and the nonrelativistic theory (quantum theory), which constitutes the foundation of all solid-state science. How does one resolve this paradox? Logic clearly indicates that it is necessary to turn to the notion of actual aether medium but 
taking into account contemporary knowledge. (Spectacular proof is provided by the physics of elementary particles in which various bag models, bubble models, lattice models, string models, etc., are paramount important. It is very difficult to consider these subquantum media merely as implicit forms.)

In the proposed theory (this paper and Ref. 14) the notion of a discrete vacuum medium is original. Hence this medium allows the creation of the the causal dynamics of an elementary particle and obtaining the equation of a particle path (15). However, there are the problem of conforming the peculiarities of the vacuum medium with special relativity. It should be noted that we already proceeded from the relativistic Lagrangian invariant (2), and it allowed us to obtain the Schrödinger equation in the invariant form (39). Furthermore, the reduction in size of the particle and superparticles as well as the availability of the adiabatic motion of the former are also related to the peculiarities of the medium.

By definition, ${ }^{(14)}$ the mass of the particle as a local curvature in the vacuum medium is the ratio between the superparticle volume $V$ in the degenerate, that is, in the nondeformed, vacuum and the particle volume $V_{0}$, that is, $M_{0}=$ const $V / V_{0}$. As the particle moves along the $X$-axis, its reduction in size by a factor of $\sqrt{1-v_{0}^{2} / c^{2}}$ in this direction automatically leads to an increase in its mass by a factor of $\left(1-v_{0}^{2} / c^{2}\right)^{-1 / 2} ; \quad$ indeed, as $M_{0} \propto V_{0}^{-1}=\left(R_{0 x} R_{0 y} R_{0 z}\right)^{-1}$, then

$$
M=M_{0} / \sqrt{1-v_{0}^{2} / c^{2}} \propto 1 /\left[\left(R_{0 x} \sqrt{1-v_{0}^{2} / c^{2}}\right) R_{0 y} R_{0 z}\right]
$$

where $R_{0 i}$ is the typical size of the particle in the state of rest along the axes $i=\{X, Y, Z\}$.

Nonetheless, the result (40) may be obtained from purely physical consideration, namely, since the motion of the particle together with the surrounding deformation coat is regarded as the travel of an element of a liquid in hydrodynamics; the appropriate equation takes the form ${ }^{(34)}$

$$
\rho d \vec{v} / d t=-\nabla \mathcal{P}
$$

where $\rho$ is the liquid element density and $\mathcal{P}$ is the pressure of the liquid on the moving element. We assume that the motion is adiabatic, that is, the change in pressure on the side of the liquid upon the element chosen is proportional to the variation in density of this element and then ${ }^{(35)}$

$$
(\partial \mathcal{P} / \partial \rho)_{\text {entropy }}=c^{2}
$$

where $c$ is the maximum velocity for this liquid (sound velocity). Equation (41) is nonlinear and consequently allows for multiple solutions. However, there is only one possibility when this equation becomes linear, and, therefore, there is a single solution. That situation is realized for our medium where the motion is highly particular.

In hydrodynamics, the point is limited by the proper size of the element of the liquid. This size is enormous as compared with the particle size. Hence the character of change of the substantial derivative $d \vec{v} / d t$ in Eq. (41) should 
be specified by microscopic processes which take place in the vacuum medium when the particle moves. The main peculiarity that results from these processes is nonstationary motion of the element of our liquid: from the initial moment of the motion its velocity changes from $v_{0}$ to 0 , then from 0 to $v_{0}$, etc., with each time interval $T / 2$. Thus a tangible change in the velocity of the element is observed over the time interval $T / 2$ and spatial interval $\lambda / 2$. The smallest scale does not appear at the continuous consideration of the motion of that liquid's element. For this case the substantial derivative may be defined as

$$
\frac{d f(x)}{d x}=\lim _{\Delta x \rightarrow\left\{\begin{array}{l}
T / 2 \\
\lambda / 2
\end{array}\right\}} \frac{f(x+\Delta x)-f(x)}{\Delta x}=\lim _{\Delta x \rightarrow\left\{\begin{array}{c}
T / 2 \\
\lambda / 2
\end{array}\right\}^{\frac{\Delta f}{\Delta x}}}
$$

In this connection, continual Eq. (41), with regard for (42), necessitates a substitution for its discrete analogy:

$$
\rho \Delta v / \Delta t=-c^{2} \Delta \rho / \Delta l
$$

here, $\Delta l=\lambda / 2$ and $\Delta t=T / 2$ are the spatial and time intervals within which the element velocity decreases from the initial value of $v_{0}$ to the final 0 , that is, $\Delta v=-v_{0}$. When the speed of the element changes from the maximum to the minimum magnitude $\left(v_{0} \rightarrow 0\right)$, the pressure, on the contrary, changes from the minimum $(\mathcal{P})$ to the maximum $\left(\mathcal{P}_{0}\right)$ magnitude; therefore, $\Delta \mathcal{P}=\mathcal{P}-\mathcal{P}_{0}$. Then, from the formula $\Delta \mathcal{P} / \Delta \rho=c^{2}$ (42) one finds that $\Delta \rho=\rho-\rho_{0}$ where $\rho$ and $\rho_{0}$ are the density of the liquid element at the moment of motion and in the state of rest, respectively. Hence, from (44), with regard to the correlation $\lambda / T=v_{0}$, we gain

$$
\rho=\rho_{0} /\left(1-v_{0}^{2} / c^{2}\right) .
$$

At the next stage of element motion the parameters of Eq. (44) are as follows: $\Delta l=\lambda / 2, \Delta t=T / 2, \Delta v=v_{0}$, and $\Delta \rho=\rho_{0}-\rho$; and again one obtains expression (45). This expression is true even at $v_{0} \rightarrow c$.

Our liquid element is essentially the deformation coat that surrounds the particle. Since the coat is a linear response of the vacuum medium to the particle creation, then the total coat mass should be equal to the particle mass, that is, to $M_{0}$ in the state of rest. This is apparently true for the volume $V_{0}$, because, according to our hypothesis, the mass of the particle is equivalent, with accuracy to the constant factor, to its inverse proper volume. The value $V_{0}$ for the element plays the role of an effective volume, but for an arbitrary element of the degenerate vacuum (where there is no particle) that the effective volume is absent, $V_{0}=0$.

So, we have for the liquid element $M_{0} \propto 1 / V_{0}$ and, therefore, $\rho_{0} \propto 1 / V_{0}^{2}$. In agreement with (45), the motion of the element with the velocity $v_{0}$ results in a decrease of the total volume of the element in the direction of the motion: $\rho_{0} \rightarrow \rho \propto 1 /\left(V_{0} \sqrt{1-v_{0}^{2} / c^{2}}\right)^{2}$. Then the mass of the element and the mass of the particle as well (due to the interdependence of these masses) change:

$$
M_{0} \propto 1 / V_{0} \rightarrow 1 /\left(V_{0} \sqrt{1-v_{0}^{2} / c^{2}}\right)
$$


that is, the mass increases in the direction of the velocity vector by a factor of $1 / \sqrt{1-v_{0}^{2} / c^{2}}$ which is likely to be in complete agreement with the experimental fact and formalism of special relativity.

\section{Conclusion}

The present concept is based on the availability of a discrete vacuum medium. This medium (quantum aether) is not contradictory to special relativity because the model: (1) satisfies Einstein's postulate of the universal speed $c$ in a vacuum, that is, the postulate corresponds to the adiabatic nonstationary motion of a material object in the aether; (2) the model correctly describes the increase of mass and reduction of dimensions of a moving object along a path. In this respect the model complies with the Lorentz hypothesis on the change of the geometry of a particle in motion. (3) The model introduces proper time, that is Lorentz-invariant, for the description of behavior of material objects.

The theory proposed here and in Ref. 14 and here sheds some light on the hidden dynamics of free moving spinless canonical particle. The theory is characterized by short-range action and is valid both for the nonrelativistic and relativistic particle. It is causal and therefore contains the equation of path [see (15)]. Moreover, this theory give rise to the basic relations of quantum mechanics [see (35) and (36)], and its outcome is the Schrödinger wave equation in a relativistic-invariant form [see (37) and (39)]. Thereby, one can affirm that the contemporary nonrelativistic quantum theory is really the relativistic one [it is only necessary that all masses $M_{0}$ in any system under consideration are replaced by relativistic masses, $M_{0} \rightarrow M_{0} / \sqrt{1-v_{0}^{2} / c^{2}}$, but this is a negligible correction with accuracy to $v_{0}^{2} / c^{2}$ since $v_{0} \ll c$ ].

Consequently, all these results allow us to regard the vacuum in the form of an elastic discrete medium and in this case inertons as elementary excitations of this medium should exist and manifest themselves experimentally.

\section{Acknowledgment}

I am very grateful to Professor Dirk ter Haar for interest in and support of this work. 


\section{APPENDIX}

For the variables $X_{(r)}^{s}$ and $x_{(r)}^{s}$ one obtains from Eq. (5) the equations of extremals:

$$
\begin{aligned}
& \ddot{X}_{(r)}^{s}+ \Gamma_{i j}^{s} \dot{X}_{(r)}^{i} \dot{X}_{(r)}^{j}+\frac{\pi}{T_{(r)}} g^{s k}\left(\frac{\partial \hat{B}_{i j}^{(r)}}{\partial X_{(r)}^{k}}\right) \\
& \times\left(X_{(r)}^{i} \dot{x}_{(r)}^{j}+\left.\dot{X}_{(r)}^{i}\right|_{t_{(r)}=0} x_{(r)}^{j}\right)+\frac{\pi}{T_{(r)}} g^{s k} \hat{B}_{k j}^{(r)} \dot{x}_{(r)}^{j}=0 ; \\
& \ddot{x}_{(r)}^{s}+\widetilde{\Gamma}_{i j}^{(r) s} \dot{x}_{(r)}^{i} \dot{x}_{(r)}^{j}+\frac{\pi}{T_{(r)}} \widetilde{g}^{(r) s k}\left(\frac{\partial \hat{B}_{i j}^{(r)}}{\partial x_{(r)}^{k}}-\frac{\partial \hat{B}^{(r)}}{\partial x_{(r)}^{j}}\right) X_{(r)}^{i} \dot{x}_{(r)}^{j} \\
& \\
& \quad-\frac{\pi}{T_{(r)}} \widetilde{g}^{(r) s k} \hat{B}_{k i}^{(r)}\left(\dot{X}_{(r)}^{i}-\left.\dot{X}_{(r)}^{i}\right|_{t_{(r)}=0}\right)=0 ;
\end{aligned}
$$

here, $\Gamma_{i j}^{s}$ and $\widetilde{\Gamma}_{i j}^{(r) s}$ are symmetrical connections (see Ref. 19) for the particle and for the $r$ th inerton, respectively; indices $i, j$, and $s$ take the values $1,2,3$. The designation

$$
\hat{B}_{i j}^{(r)} \equiv\left(g_{i f}\left(\hat{A}^{-1} \widetilde{g}_{f j}^{(r)}\right)_{o}\right)^{1 / 2}
$$

is introduced into (A1) and (A2). The process of "knocking out" of the next $r$ th inerton can be subdivided into three stages (Ref. 14). At the first stage the interaction operator in Eqs. (A1) and (A2) is still not engaged, $\hat{B}_{i j}^{(r)}=0$. Then the termwise difference between these equations is reduced to the form

$$
\left(\ddot{X}_{(r)}-\ddot{x}_{(r)}\right)+\left(\Gamma_{i j}^{s} \dot{X}_{(r)}^{i} \dot{X}_{(r)}^{j}-\widetilde{\Gamma}_{i j}^{(r) s} \dot{x}_{(r)}^{i} \dot{x}_{(r)}^{j}\right)=0 .
$$

Equation (A4) describes the union of the particle and $r$ th inerton into a common system, and because of this, the acceleration that one of the partners of the system experiences coincides with the acceleration that other partner experiences. Hence the difference in the first set of parentheses in Eq. (A4) is equal to zero and one gains the relation

$$
\Gamma_{i j}^{s} \dot{X}_{(r)}^{i} \dot{X}_{(r)}^{j}=\widetilde{\Gamma}_{i j}^{(r) s} \dot{x}_{(r)}^{i} \dot{x}_{(r)}^{j} .
$$

Coefficients $\Gamma_{i j}^{s}$ are generated by the particle mass $M_{o}$, but coefficients $\widetilde{\Gamma}_{i j}^{(r) s}$ are generated by the inerton mass $m_{r}$ (see Ref. 14). Hence the relation $\Gamma_{i j}^{s} / \widetilde{\Gamma}_{i j}^{(r) s}=$ $M_{o} / m_{r}$ holds. If the velocity of the particle at the point of emission of the $r$ th inerton is $v_{o r}$ and the initial velocity of this emitted inerton is $c$, then one obtains

$$
M_{0} v_{0 r}^{2}=m_{r} c^{2} .
$$


Setting tensors $g_{i j}$ and $\widetilde{g}_{i j}^{(r)}$ are constant (see Ref. 14), one can reduce Eqs. (A1) and (A2) to the form when only two terms remain: the first and the last ones, the latter being transformed as follows:

$$
\begin{gathered}
g^{s k} \hat{B}_{k j}^{(r)} \rightarrow\left(m_{r} / M\right)^{1 / 2}=v_{0 r}^{s} / c \\
\widetilde{g}^{(r) s k} \hat{B}_{k j}^{(r)} \rightarrow\left(M / m_{r}\right)^{1 / 2}=c / v_{0 r}^{s} .
\end{gathered}
$$

As the result, Eqs. (A1) and (A2) take the form (one omits the parentheses for indices $r$ ):

$$
\begin{gathered}
\ddot{X}_{r}^{s}+\frac{\pi}{T_{r}} \frac{v_{o r}}{c} \dot{x}_{r}^{s}=0 \\
\ddot{x}_{r}^{s}-\frac{\pi}{T_{r}} \frac{c}{v_{o r}}\left(\dot{X}_{r}^{s}-v_{o r}^{s}\right)=0 .
\end{gathered}
$$

Initial conditions are chosen in the form

$$
\left.\dot{X}_{r}\left(t_{r}+\Delta t_{r}\right)\right|_{t_{r}=0}=\dot{X}\left(\Delta t_{r}\right)=v_{0 r}
$$

for the particle and

$$
\left.x^{\perp}\right|_{t_{r}=0}=0,\left.\quad \dot{x}^{\perp}\right|_{t_{r}=0}=c
$$

for the $r$ th inerton.

The solution of Eqs. (A9) and (A10) is entirely similar to the solution of the equations of motions carried out previously ${ }^{(14)}$ in the nonrelativistic limit. 


\section{References}

1. W. Pauli, Scientia (Milan) 56, 65 (1936).

2. L. de Broglie, Ann. Fond. L. de Broglie 12, 399 (1987).

3. J.S. Bell, Speakable and Unspeakable in Quantum Mechanics (Cambridge University Press, Cambridge, 1987).

4. A.O. Barut and M. Bozic, Ann. Fond. L. de Broglie 15, 67 (1990).

5. C.L.B. Ryff, Found. Phys, 20, 1061 (1990).

6. M. Yusouff, Preprint IC/83/46, Int. Centre Theor. Phys. (Miramare -Trieste, 1983).

7. P. Multanrzynski and M. Heller, Found. Phys. 20, 1005 (1990).

8. C. Wolf, Ann. Fond. L. de Broglie 15, 189 (1990).

9. K.E. Plokhotnikov, Doklady Acad. Nauk USSR 316, 332 (1991) (in Russian).

10. G. Brightwell and R. Gregory, Phys. Rev. Lett. 66, 260 (1991).

11. C.W. Rietdijk, Ann. Fond. L. de Broglie 16, 177 (1991).

12. P.I. Fomin, in Quantum Gravity. Proc. IV Seminar on Quantum Gravity, Moscow, USSR, 25 - 29 May 1987; Eds. V. Markov, V. Berezin, and V.P. Frolov (World Scientific Publishing Co., Singapore, 1988), p. 813.

13. F. Winterberg, Int. J. Theor. Phys. 34, 265 (1995).

14. V. Krasnoholovets and D. Ivanovsky, Phys. Essays 6, 554 (1993) (also e-preprint xxx.lanl.gov quant/9910023).

15. B.A. Dubrovin, S.P. Novikov and A.T. Fomenko, Modern Geometry (Nauka, Moscow, 1986), p. 24-36 (in Russian).

16. L.D. Landau and E.M. Lifshits, The Theory of Field (Nauka, Moscow, 1973), p. 42 (in Russian).

17. B.A. Dubrovin, S.P. Novikov and A.T. Fomenko, Modern Geometry (Nauka, Moscow, 1986), p.295 (in Russian).

18. Ibid., p. 291.

19. Ibid., pp. $289-291$.

20. D. ter Haar, Elements of Hamiltonian Mechanics (Nauka, Moscow, 1974), p. 157 (Russian translation). 
21. H. Goldstein, Classical Mechanics (Nauka, Moscow, 1975), p. 306 (Russian translation).

22. L. de Broglie, Heisenberg's Uncertainty Relations and the Probabilistic Interpretation of Wave Mechanics (Mir, Moscow, 1986), pp. 42-43 (Russian translation).

23. E. Fermi, Notes on Quantum Mechanics (Mir, Moscow, 1965), pp. 15-19 (Russian translation).

24. L.I. Schiff, Quantum mechanics (Izdatelstvo Inostrannoy Literatury, Moscow, 1959), p. 33 (Russian translation).

25. Ibid., p. 364 .

26. A.A. Sokolov, Yu.M. Loskutov and I.M. Ternov, Quantum Mechanics (Prosveshchenie, Moscow, 1965), p.55 (in Russian).

27. L. de Broglie, Heisenberg's Uncertainty Relations and the Probabilistic Interpretation of Wave Mechanics (Mir, Moscow, 1986), pp. 34-42 (Russion translation).

28. B.A. Dubrovin, S.P. Novikov and A.T. Fomenko Modern Geometry (Nauka, Moscow, 1986), p.63 (in Russian).

29. P.G. Bergmann, Introduction to the Theory of Relativity (Gosudarstvennoe Izdatelstvo Inostrannoy Literatury, Moscow, 1947), pp. 72-73, 114-115 (Russian translation).

30. H.A. Lorentz, Aether Theories and Aether Models (NauchnoTekhnicheskoe Izdatelstvo NKTP USSR, Moscow - Leningrad, 1936), p. 25 (Russian translation).

31. Idem, Amst. Proc., 6, 809 (1904); 12, 986 (1904).

32. P. Ehrenfest, Zhurn. Russkago Fiz.-Khim. Obshchestva (St.-Petersburg), Part Phys., 45, No. 4B, 151 (1913).

33. A. Einstein, Ann. d. Phys., B 17, Ser. 4, 891 (1905).

34. L.D. Landau and E.M. Lifshits, Hydrodynamics (Nauka, Moscow, 1986), p. 15 (in Russian).

35. Ibid., p. 351. 